\title{
Subjective health definition in persons suffering from schizophrenia
}

Beata Dutczak, Leszek Bidzan

\section{Source}

Beata Dutczak, Leszek Bidzan. (2010). Subjective health definition and health behaviors in

persons suffering from schizophrenia. Ann Gen Psychiatry, vol. 9 (S1)

Patients suffering from schizophrenia define health as ability to cooperate with other persons, to enjoy life and to control their feelings, emotions and vehemence. 